\title{
Application of the FUN3D Unstructured-Grid Navier-Stokes Solver to the 4th AIAA Drag Prediction Workshop Cases
}

\author{
E. M. Lee-Rausch * D. P. Hammond ${ }^{\dagger} \quad$ E. J. Nielsen ${ }^{\ddagger} \quad$ S. Z. Pirzadeh ${ }^{\S} \quad$ C. L. Rumsey $\rrbracket$ \\ NASA Langley Research Center, Hampton, VA 23681
}

\begin{abstract}
FUN3D Navier-Stokes solutions were computed for the 4th AIAA Drag Prediction Workshop grid convergence study, downwash study, and Reynolds number study on a set of node-based mixed-element grids. All of the baseline tetrahedral grids were generated with the VGRID (developmental) advancing-layer and advancing-front grid generation software package following the gridding guidelines developed for the workshop. With maximum grid sizes exceeding 100 million nodes, the grid convergence study was particularly challenging for the node-based unstructured grid generators and flow solvers. At the time of the workshop, the super-fine grid with 105 million nodes and 600 million elements was the largest grid known to have been generated using VGRID. FUN3D Version 11.0 has a completely new pre- and post-processing paradigm that has been incorporated directly into the solver and functions entirely in a parallel, distributed memory environment. This feature allowed for practical pre-processing and solution times on the largest unstructured-grid size requested for the workshop. For the constant-lift grid convergence case, the convergence of total drag is approximately second-order on the finest three grids. The variation in total drag between the finest two grids is only 2 counts. At the finest grid levels, only small variations in wing and tail pressure distributions are seen with grid refinement. Similarly, a small wing side-of-body separation also shows little variation at the finest grid levels. Overall, the FUN3D results compare well with the structured-grid code CFL3D. The FUN3D downwash study and Reynolds number study results compare well with the range of results shown in the workshop presentations.
\end{abstract}

\section{Introduction}

The fourth international AIAA Drag Prediction Workshop (DPW-IV) was held in San Antonio, Texas in June 2009 to evaluate CFD transonic cruise drag predictions for subsonic transport aircraft. ${ }^{1}$ The objectives of the workshop were as follows: 1) to build on the success of past AIAA Drag Prediction Workshops, ${ }^{2-4}$ 2) to assess the state-of-the-art computational methods as practical aerodynamic tools for aircraft force and moment prediction of industry relevant geometries, 3) to provide an impartial forum for evaluating the effectiveness of existing computer codes and modeling techniques using Navier-Stokes solvers, and 4) to identify areas needing additional research and development. The focus of this particular workshop was on "blind" drag prediction (a priori experimental data was not available for comparison) for the NASA Common Research Model (CRM) transonic wing-body-tail configuration. ${ }^{5}$ As with prior Drag Prediction Workshops, grid convergence of the force and moments coefficients was evaluated at the design cruise Mach number and lift coefficient. With maximum grid sizes exceeding 100 million nodes (600 million cells), the grid convergence study was particularly challenging for the node-based unstructured grid generators and flow solvers. This maximum grid size was four times larger than required for DPW-III. Additionally in this workshop, the drag increment due to the trimming of the aircraft and drag increment due to Reynolds number scaling were evaluated. An optional Mach sweep study was also requested to look at drag rise.

\footnotetext{
${ }^{*}$ Senior Member AIAA, Research Engineer.

${ }^{\dagger}$ Senior Research Scientist.

${ }^{\ddagger}$ Senior Member AIAA, Senior Research Scientist.

$\S$ Senior Member AIAA, Senior Research Engineer (Posthumous).

${ }^{\top}$ Associate Fellow AIAA, Senior Research Scientist.
} 
The experimental data for the CRM and the statistical analysis of the workshop results are scheduled for publication in June 2010 ${ }^{6,7}$ As with previous DPW workshop, the DPW-IV test cases will in all likelihood become standard CFD test cases. Eliasson and Peng have already published a paper looking at the influence of turbulence modeling and grid resolution in computations of the CRM. ${ }^{8}$ Hashimoto et al. have used the CRM to evaluate a hexahedra grid generation method. ${ }^{9}$ Many more computational studies of the DPW-IV test cases are scheduled for publication at the 28th AIAA Applied Aerodynamics Conference in June 2010.

Results from the DPW-IV grid convergence study, downwash study, and Reynolds number study were submitted to the workshop for the FUN3D ${ }^{10-12}$ unstructured-grid Navier-Stokes flow solver on a set of node-based mixed-element grids. This paper gives a summary of the FUN3D results submitted to the workshop. A description of the unstructured node-based grids is provided in this paper detailing the grid parameters and spacings. Some improvements to the FUN3D solver/grid processing efficiency and parallelization are also discussed. These improvements allowed for the practical processing of the largest unstructured-grid sizes requested for the workshop (100 million nodes). Although experimental data are not yet available, comparisons are made with overset structured grid results from the well-known code CFL3D. ${ }^{13}$

\section{DPW-IV Workshop Cases}

All of the DPW-IV workshop cases are based on the analysis of the NASA CRM wing/body/tail configuration shown in Figure 1. The configuration is a transonic transport aircraft designed to fly at a cruise Mach number of $M_{\infty}=$ 0.85 with a nominal lift condition of $C_{L}=0.500$ at a Reynolds number of $R e_{c}=40 \times 10^{6}$ per mean aerodynamic chord $\left(c_{r e f}\right)$. The reference geometric quantities for the CRM are summarized in Table II. A detailed description of the CRM can be found in Ref. 5. The first test case (Case 1) for the workshop included a grid convergence study and a downwash study. For the grid convergence study, computations were performed for a constant design-lift coefficient of $C_{L}=0.500 \pm 0.001$ at a freestream Mach number of $M_{\infty}=0.85$ and freestream chord Reynolds number of $R e_{c}=5 \times 10^{6}$. (Note that this Reynolds number represented a planned wind tunnel test condition.) For the grid convergence study, a nominal tail incidence angle $i_{h}=0^{\circ}$ was specified. A family of four grids was required for the study with consistent levels of refinement between each grid: coarse (3.5 million nodes), medium (10 million nodes), fine (35 million nodes), and super-fine (100 million nodes). The downwash study required complete angle-of-attack sweeps for the CRM with three different tail settings $\left(i_{h}=-2^{\circ}, 0^{\circ},+2^{\circ}\right)$ on a set of medium sized grids. The trimmed drag polar was then derived from an interpolation of the polars at the variable tail incidences using a process provided by the organizing committee. The delta drag polar was then computed by differencing the polar results from a tail-off configuration with results from the trimmed tail-on drag polar. The optional Reynolds number study (Case 3) is a comparison of $R e_{c}=5 \times 10^{6}$ results with $R e_{c}=20 \times 10^{6}$ results on the medium grid size at the design Mach number and lift condition $\left(M_{\infty}=0.85\right.$ and $\left.C_{L}=0.500 \pm 0.001\right)$. For Case 3, the nominal tail incidence angle $i_{h}=0^{\circ}$ was used. FUN3D results were not computed for the optional Mach sweep study (Case 2).

Table 1. Reference geometry for the CRM.

\begin{tabular}{|l|r|}
\hline Mean Aerodynamic Chord $\left(c_{r e f}\right)$ & 275.80 inch \\
\hline Wing Reference Area/2 & 297,360 inch $^{2}$ \\
\hline Wing Span/2 & 1159.0 inch \\
\hline Aspect Ratio $(A R)$ & 9.0 \\
\hline
\end{tabular}

\section{DPW-IV Unstructured LaRC Node-Based Grids}

The FUN3D solutions were computed using a mixed-element version of the vertex-based unstructured tetrahedral grids generated for the workshop by Dr. Pirzadeh. The tetrahedral grids are available on the DPW-IV web site under the label "unstructured LaRC/NodeBase." "All of the baseline tetrahedral grids were generated with the VGRID (developmental) advancing-layer and advancing-front grid generation software package ${ }^{14}$ following the gridding guidelines developed for the workshop. ${ }^{1}$ The grids generated with VGRID were fully tetrahedral. However, VGRID uses an advancing layer technique to generate the boundary layer portion of the grid so that prisms can be reconstructed in the boundary layer for use with a mixed-element discretization. In the boundary layer, three tetrahedral cells are com- 
bined into one prism. The mixed-element grids have essentially the same number of nodes and nodal spacing as the fully tetrahedral grids although the number of cells and the shape of the control volumes differ in the boundary layers. The merging process adds a trivial number of node points in the interface region between the advancing layers and advancing front to construct a valid topology in the interface region between the prismatic layers and the tetrahedral region. For the LaRC node-based grids, the merging process also reduces the total number of cells in the grid by a factor of approximately $1 / 2$ to $2 / 3$.

For the grid convergence study, a set of four globally-refined grids were generated following the gridding guidelines. Similarly, for the downwash study, a set of three consistently sized grids with varying tail incidences were generated. For the tail-off configuration, the tail solid surfaces were removed, and the volume grid was re-generated with the same spacing requirements in the location of the tail as were used in the tail-on configuration. A summary of the Case 1 mixed-element grid sizes and runs is shown in Table III. For the Case 3 high Reynolds number grid, the surface grid distribution from the Case 1 medium grid was used with a smaller minimum wall spacing. The total number of grid nodes for the $R e_{c}=20 \times 10^{6}$ grid was 11 million. Note that at the time of the workshop, the Case 1 super-fine grid with 105 million nodes and 600 million elements was the largest grid known to have been generated using VGRID.

Table 2. Summary of DPW-IV Case 1 mesh sizes and runs at $M_{\infty}=0.85, R_{c}=5 \times 10^{6}$.

\begin{tabular}{|l|r|r|r|r|}
\hline Grid & Tail Incidence (deg.) & Grid Size (million nodes) & $C_{L}=0.500$ & Alpha Polar \\
\hline \hline Coarse & 0 & 3.7 & required & not required \\
\hline Medium & 0 & 10.3 & required & required \\
\hline Fine & 0 & 35.5 & required & not required \\
\hline Super Fine & 0 & 105 & required & not required \\
\hline Medium & -2 & 10.3 & not required & required \\
\hline Medium & +2 & 10.3 & not required & required \\
\hline Medium & No Tail & 8.2 & not required & required \\
\hline
\end{tabular}

VGRID has two types of spacing requirements: the "inviscid" spacing distributions are used in the advancingfront region of the mesh, and the "viscous" spacing distributions are used in the advancing-layer regions of the mesh where high stretching is required. The different grids for the grid refinement study were generated by a global coarsening/refinement of the inviscid spacing parameters (VGRID "sources") and a global coarsening/refinement of the viscous wall spacing. A summary of characteristic grid spacing for the wing/body/tail $\left(i_{h}=0^{\circ}\right)$ configuration is shown in Table 3 to illustrate that the grids were generated following the gridding guidelines for the workshop. The global spacing factor shown in Table 3 represents the relative coarsening/refinement of the inviscid spacing parameters for each of the grids. The range of chordwise spacing for the wing and tail leading edge (LE) and trailing edge (TE) fall close to the $0.1 \%$ local chord specified for the medium grids at the root chord $\left(c_{\text {root }}\right)$ and tip chord $\left(c_{t i p}\right)$ locations. The spanwise stretching of the grids was 10:1 near the wing LE and 15:1 near the wing TE. There were similar limits on the spanwise stretching near the tail LE and TE. The limits on the grid spanwise stretching rates keep the wing and tail spanwise spacing at root and tip well within the workshop requirement $(0.1 \%$ local semi-span for the medium grid). A composite view of the medium grid wing/body/tail grid is shown in Figure 2 to illustrate the overall topology of the surface mesh.

The clustering of points normal to the surface was computed according to the VGRID stretching function ${ }^{15}$

$$
\delta_{n}=\delta_{1}\left[1+r_{1}\left(1+r_{2}\right)^{n-1}\right]^{n-1}
$$

where $\delta_{n}$ is the normal spacing of the $n^{t h}$ layer, $\delta_{1}$ is the spacing of the first layer, and the factors $r_{1}$ and $r_{2}$ are constants that determine the rate of stretching. (Note if $r_{2}$ is zero the stretching is geometric.) Based on the advancing layer stretching factors given in Table 3, the maximum growth rate of the cell sizes in the viscous layers is approximately 1.19 which is less than the 1.25 requested in the gridding guidelines. The blunt trailing edges of the wing and nacelles were resolved in all grids by explicitly dividing the trailing-edge surfaces into the specified number of cells. 
Table 3. Summary of characteristic grid spacing for the DPW-IV Unstructured LaRC Node-Based wing/body/tail $i_{h}=0^{\circ}$ grids.

\begin{tabular}{|l|l|l|l|l|}
\hline & \multicolumn{4}{|c|}{ Node-Centered Grids } \\
\hline & Coarse & Medium & Fine & Super Fine \\
\hline \hline Global Spacing Factor & 1.654 & 1.00 & 0.562 & 0.355 \\
\hline \% Chordwise Spacing & $0.20 \% c_{\text {root }}$ & $0.12 \% c_{\text {root }}$ & $0.07 \% c_{\text {root }}$ & $0.04 \% c_{\text {root }}$ \\
at wing LE & $0.20 \% c_{\text {tip }}$ & $0.12 \% c_{\text {tip }}$ & $0.07 \% c_{\text {tip }}$ & $0.04 \% c_{\text {tip }}$ \\
\hline$\%$ Chordwise Spacing & $0.07 \% c_{\text {root }}$ & $0.04 \% c_{\text {root }}$ & $0.02 \% c_{\text {root }}$ & $0.01 \% c_{\text {root }}$ \\
at wing TE & $0.12 \% c_{\text {tip }}$ & $0.07 \% c_{\text {tip }}$ & $0.04 \% c_{\text {tip }}$ & $0.02 \% c_{\text {tip }}$ \\
\hline$\%$ Chordwise Spacing & $0.20 \% c_{\text {root }}$ & $0.16 \% c_{\text {root }}$ & $0.09 \% c_{\text {root }}$ & $0.06 \% c_{\text {root }}$ \\
at tail LE & $0.26 \% c_{\text {tip }}$ & $0.12 \% c_{\text {tip }}$ & $0.07 \% c_{\text {tip }}$ & $0.04 \% c_{\text {tip }}$ \\
\hline$\%$ Chordwise Spacing & $0.20 \% c_{\text {root }}$ & $0.12 \% c_{\text {root }}$ & $0.07 \% c_{\text {root }}$ & $0.04 \% c_{\text {root }}$ \\
at tail TE & $0.20 \% c_{\text {tip }}$ & $0.12 \% c_{\text {tip }}$ & $0.07 \% c_{\text {tip }}$ & $0.04 \% c_{\text {tip }}$ \\
\hline Wing/Tail TE Cells & $8 / 8$ & $12 / 12$ & $16 / 16$ & $24 / 24$ \\
\hline Spacing Fuselage Nose/Aft Body & $4.0 \% c_{\text {ref }}$ & $2.4 \% c_{\text {ref }}$ & $1.3 \% c_{\text {ref }}$ & $0.9 \% c_{\text {ref }}$ \\
\hline Average Cell $y^{+}$ & 1.0 & 0.667 & 0.444 & 0.296 \\
\hline Nominal Prism & 25 & 25 & 25 & 25 \\
Layer Cells & & & & \\
\hline Viscous Wall & 0.001478 & 0.0009850 & 0.0006570 & 0.0004380 \\
Spacing $\delta_{1}($ inches $)$ & & & & \\
\hline Viscous Stretching & 0.12, & 0.12, & 0.12, & 0.12, \\
$r_{1}, r_{2}$ & 0.02 & 0.02 & 0.02 & 0.02 \\
\hline Outer Boundary Box & $100 c_{\text {ref }}$ & $100 c_{\text {ref }}$ & $100 c_{\text {ref }}$ & $100 c_{\text {ref }}$ \\
\hline
\end{tabular}

\section{FUN3D Flow Solver}

FUN3D ${ }^{10-12}$ is a finite-volume Reynolds-averaged Navier-Stokes (RANS) solver in which the flow variables are stored at the vertices or nodes of the mesh. FUN3D solves the equations on mixed element grids, including tetrahedra, pyramids, prisms, and hexahedra and also has a two-dimensional path for triangular/quadrilateral grids. It employs an implicit upwind algorithm in which the inviscid fluxes are obtained with a flux-difference-splitting scheme. At interfaces delimiting neighboring control volumes, the inviscid fluxes are computed using an approximate Riemann solver based on the values on either side of the interface. Several convective flux schemes are available in FUN3D. The most common scheme for subsonic and transonic flows is Roe's flux difference splitting ${ }^{16}$ which is used in the current study. For second-order accuracy, interface values are obtained by extrapolation of the control volume centroidal values, based on gradients computed at the mesh vertices using an unweighted least-squares technique. Several flux limiters are available in FUN3D to limit the reconstructed values when necessary. The most common used for transonic flows is that of Venkatakrishnan ${ }^{17}$ which is used in this study. For tetrahedral meshes, the full viscous fluxes are discretized using a finite-volume formulation in which the required velocity gradients on the dual faces are computed using the Green-Gauss theorem. On tetrahedral meshes this is equivalent to a Galerkin type approximation. For non-tetrahedral meshes, the same Green-Gauss approach can lead to odd-even decoupling. A pure edge-based approach can be used to circumvent the odd-even decoupling issue, but yields only approximate viscous terms. Thus for non-tetrahedral meshes, the edge-based gradients are combined with Green-Gauss gradients, which improves the h-ellipticity of the operator, and allows the complete viscous stresses to be evaluated. ${ }^{18,19}$ This formulation results in a discretization of the full Navier-Stokes equations.

The solution at each time-step is updated with a backwards Euler time-differencing scheme. At each time step, the linear system of equations is approximately solved with either a multi-color point-implicit procedure or an implicitline relaxation scheme. ${ }^{20}$ Local time-step scaling is employed to accelerate convergence to steady-state. For turbulent flows, several models are available within FUN3D. The most common are the one-equation model of SpalartAllmaras $^{21}$ (S-A) and the two-equation shear stress transport (SST) model of Menter. ${ }^{22}$ The S-A model may be solved 
loosely coupled to the mean-flow equations or tightly coupled to the mean-flow equations. The Menter SST Model ${ }^{22}$ is loosely coupled. The loosely coupled S-A model is used for the current study.

Efforts were initiated in the summer of 2006 to improve the computational performance of the FUN3D code. Many low-level aspects of the code were examined including cache reuse, MPI communication, inlining, basic blocks and alternative ordering techniques for the grid/linear algebra operations. The result of this effort was a factor of 6.5 speed-up of the recently-released Version 11.0 over Version 10.3.1 (May 2006). The code performance at several points in time from May 2006 to the the current Version 11.0 is shown in Figure 3(a). The comparison of density residual versus wall clock time shows a 6.5 times speed-up for a wing/body transonic Navier-Stokes solution to the same level of residual convergence. This timing study was performed with fixed compiler, compiler options, computer hardware and solver options so that all of the code speed-up shown is due to computer science and implementation differences. The FUN3D solver has also demonstrated linear scaling in parallel efficiency up to 8,192 computer cores on the Pleiades supercomputer at the NASA Advanced Supercomputing (NAS) facility. Pleiades is an SGI ICE system with Xeon E5472 and X5570 processor cores. The scaling study utilized the Xeon E5472 processors with 2 quad-core processors per node and $8 \mathrm{~GB}$ of memory per node. Figure 3(b) shows the parallel scalability of the Version 11.0 code on a range of grid sizes including the DPW-IV 105 million node super-fine mesh described in the previous section. Grids from the second DPW (DPW-II) and the Ares-1X launch vehicle are also included in Fig. 3(b). Most of the results shown were run fully-dense; namely, one process per core (e.g., eight processes on a dual-quad node). One timing study for the Ares-1X was run in a half-dense environment (four processes on a dual-quad node) to illustrate the speed-up from decreasing the inter-processor communication. The solutions for the the timing study were not run to convergence so no absolute times are provided in Fig. 3(b).

FUN3D Version 11.0 also has a completely new pre- and post-processing paradigm that has been incorporated directly into the solver and functions entirely in a parallel, distributed memory environment. The result is an end-toend scalable approach that eliminates hardware constraints as problem sizes grow. The new paradigm also cuts down on pre- and post-processing times considerably and greatly simplifies the use of FUN3D. For the 105 million node tetrahedral grid, grid partitioning and pre-processing took approximately 8 minutes using 1,024 cores on Pleiades. Partitioning the same grid on 1,024 processors using serial pre-processor previously required two weeks using 800 gigabytes (GB) of shared-memory on the NAS Columbia supercomputer which has SGI Altix 3700/4700 cores with approximately $2 \mathrm{~GB}$ of memory per core. A substantial decrease in $\mathrm{I} / \mathrm{O}$ processing time, and I/O memory overhead, was seen using either of the two new I/O options within Version 11.0 (i.e., FORTRAN stream I/O, MPI/IO).

\section{Computational Results}

The FUN3D workshop results were computed using the full Navier-Stokes equations with the flux-differencesplitting scheme of Roe, the Venkatakrishnan flux-limiter, and the loosely-coupled S-A turbulence model on mixed element grids (prisms through the boundary layer, tetrahedra in the farfield). In accordance with the workshop specifications, all computations assume fully turbulent flow. For all cases, local time-step scaling was used to accelerate the residual convergence of the non-linear equations and achieve steady-state values of forces and moments. For the constant lift cases, the angle of attack $(A O A)$ was relaxed based on the difference in total lift and the target lift during the convergence history. (Note that updating the angle of attack at every iteration slows the solution convergence compared to a case where the angle of attack is constant.) Good solution residual, force, and moment convergence was achieved for the all the workshop computations even on the super-fine grid size. Figure 4 shows the non-linear solution residual, force, and moment convergence histories for the medium and super-fine grids. The L2 norm of the density $\left(R_{1}\right)$ residual and the turbulence model $\left(R_{6}\right)$ residual are shown for each local time-step.

Total force and moment results for the CRM grid convergence study are given in Fig. 5. The angle of attack, total drag $\left(C_{D}\right)$, pressure drag $\left(C_{D p}\right)$, skin friction drag $\left(C_{D s f}\right)$ and pitching moment $\left(C_{M y}\right)$ are plotted versus $N^{-2 / 3}$, where $N$ is the number of nodes for the unstructured grid. (In the asymptotic range, one would expect a linear variation in forces or moments with $N^{-2 / 3}$ for a second order scheme for a family of three-dimensional grids that are globally refined.) Thus, results using finer grids appear to the left in the figures, and results using coarser grids appear to the right. The variation in total drag on the finest three grids is about 6 counts, and the variation on the finest two grids is 2 counts. The decomposition of the drag into pressure and skin friction components illustrates that the majority of the variation is due to the pressure component. While the changes in drag and angle of attack are nearly linear on the finest three grids, the change in pitching moment is not, possibly due to the sensitivity of the wing pitching moment to shock strength. Since the experimental data and workshop statistics are not currently available, the FUN3D results from the grid convergence study are compared to results from a well-known structured-grid code, CFL3D. ${ }^{23}$ The CFL3D results were computed on a set of overset grids using an upwind algorithm, the SA turbulence 
model, and the thin-layer approximation of the RANS equations. A complete description of the overset grids and CFL3D results can be found in Ref. 13 . The results for the CFL3D DPW-IV grid convergence study are compared with the FUN3D results in Fig. 5. Overall, the comparison between the computed forces and moments is good. The comparison in total drag between the CFL3D results on finest grid (55M cells) and the FUN3D results on the fine and super-fine grids is within 5 drag counts with most of the variation coming from the pressure component. Similarly, the comparison in pitching moment is quite good at the finest grid levels.

The component forces and moments for the wing, body and tail are shown in Fig. 6. The CFL3D results from Ref. 13 are also included for comparison. The range of the vertical scales is the same for each component of lift, drag and pitching moment which allows for a comparison of variation between the component force and moment contributions. (The data ranges for drag and pitching moment in Fig. 6 are also the same as shown in Fig. 5.) Figures 6(a)-(c) show that the lift contributions from each of the components is relatively constant with grid refinement. The drag on each of the components consistently decreases with grid refinement as shown in Figures 6(d)-(f). However, the tail variation is much less than the wing and body variation which are similar in magnitude. Figures 6(g)-(i) show that the pitching moment contributions from the body and tail are relatively constant with grid refinement with most of the variation coming from the wing. In comparing the FUN3D results to the CFL3D results, the structured grid solutions consistently have more of a download on the tail which explains the prediction of a more nose-down tail pitching moment from CFL3D. Figures 6(d)-(f) illustrate that the body and tail drag predictions are very close on the fine grids with most of the difference in total drag between the FUN3D and CFL3D results come from the wing component.

Figures 7 and 8 show the grid convergence of the wing and tail chordwise pressure coefficient $\left(C_{p}\right)$ distributions, respectively. The computational results are shown at selected stations across the wing and tail span that correspond to requested workshop and experimental data locations. (Note that the inset upper-surface pressure line contours are from the fine grid solution.) Figure 7 shows that on the inboard half of the wing the differences between the surface pressures on all the grid densities is very small. On the outboard wing, there are more differences in shock strength across the grid range, especially at the tip where the "double shock" structure does not show up at the coarsest grid density. However, the pressure distributions on the fine and super fine grids are very close. Figure 8 shows that the variation in surface pressure with grid refinement is very small across the tail with only small differences in the leading edge pressure peak. The wing and tail surface pressures from the FUN3D medium and fine grid solutions are compared to the CFL3D medium grid solutions in Fig. 9. (Note that the fine and medium grid CFL3D pressures distributions are very similar to within plotting accuracy, so only the medium grid results are shown.) The wing pressures at the mid-span location compare very well between the two codes. At the wing tip location, the overall pressure distribution compares well except near the double-shock feature, where FUN3D's two shocks are positioned slightly closer together. The tail pressures at the mid-span and tip locations also compare very well between the two codes with only a slight decrease in nose pressure on the lower surface of the tail for the CFL3D results. This difference could explain the increased tail load predicted with CFL3D.

The CRM fine grid skin friction coefficient and surface restricted streamlines shown in Figure 10 illustrate the flow patterns on the wing and tail upper surface. The flow patterns on the fine grid are indicative of those seen on the other grid densities. As was seen in DPW-II and DPW-III, there is an area of flow separation at the trailing edge of the wing-root juncture. There is also a pattern of small trailing-edge separation on the outboard portion of the wing. The grid convergence of CRM wing chordwise skin friction distribution at the $\eta=0.603$ span station shown in Figure 11 illustrates that there is a small increase in trailing-edge separation with grid refinement. The effect of grid refinement on the trailing-edge wing-root juncture flow is shown in Figure 12. In this figure, a close-up of the surface restricted streamlines on the wing upper surface is shown from a perspective just downstream and outboard of the trailing edge. The comparison of streamline patterns shows an increase in the size of the separated region with grid refinement between coarse, medium, and fine grids, but then very little change between fine and super-fine. No trailing-edge or side-of-body separation was noted on the tail at any grid density. Not all codes in the workshop predicted the same flow patterns on the CRM. ${ }^{1}$ Although some codes in the workshop predicted similar patterns of flow separation on the CRM, other codes predicted no wing-root juncture separation and/or no wing trailing-edge separation. Also some codes predicted a separation pattern on the fuselage just downstream of the tail trailing edge. Although not shown, CFL3D predicted a slightly smaller side-of-body separation region than FUN3D at the medium grid resolution, but the bubble decreased in size as the grid was refined. This may be due to the use of the thin-layer approximation in the CFL3D results. Use of the thin-layer approximation has been shown to reduce the size of the side-of-body separation in DPW-III computations. ${ }^{24}$

Figures 13 and 14 show the computational results of the downwash study. Figure 13 illustrates the effects of the tail incidence on the CRM total lift, idealized drag, and pitching moment coefficients, where idealized drag is defined as $C_{D}-C_{L}^{2} /(\pi A R)$. The CFL3D results from Ref. 13 are also included in Fig. 13 for comparison. The comparison of 
lift prediction between the two codes is quite good across the angle-of-attack and tail incidence range. Similarly, the comparison of pitching moment is also quite good except at the highest angle of attack. The idealized drag prediction from CFL3D is consistently lower than the FUN3D results across the angle-of-attack and tail incidence range except at 4 degrees angle of attack where they are very close. It is interesting to note that even when the codes do not predict the same absolute values of forces and moments the deltas due to tail incidence appear to be at the same levels. The trimmed polar forces and moments interpolated from the FUN3D results are shown in Figure 14 along with the tail-off polar data. The delta drag due to trimming the tail at the design lift coefficient $C_{L}=0.500$ is 27 counts. Range of delta drag due to trimming from the workshop presentations was $27-40$ counts. ${ }^{1}$

For the Case 3 Reynolds number study as the Reynolds number was increased to $R e_{c}=20 \times 10^{6}$, the angle of attack decreased from $2.32^{\circ}$ to $2.14^{\circ}$, and the total CRM drag dropped by 33 counts. The drag due to pressure dropped by 21 counts, and the drag due to skin friction dropped by 12 counts. The wing-root juncture separation decreased by about half the size. The range of delta drag due to increased Reynolds number from the workshop presentations was 30-35 counts. ${ }^{1}$

\section{Summary}

FUN3D Navier-Stokes solutions were successfully computed for the DPW-IV grid convergence study, downwash study, and Reynolds number study on a set of node-based mixed-element grids. All of the baseline tetrahedral grids were generated with the VGRID (developmental) advancing-layer and advancing-front grid generation software package. Although the grids generated with VGRID were fully tetrahedral, a pre-processing step was used to combine the tetrahedral cells in the boundary-layer portion of the grid into prisms. For the grid convergence study, a set of four globally refined grids were generated following the gridding guidelines. Similarly, for the downwash study, a set of three consistently sized grids with varying tail incidences were generated. For the tail-off configuration, the tail solid surfaces were removed, and the volume grid was re-generated with the same spacing requirements in the location of the tail as were used in the tail-on configuration. For the high Reynolds number grid, the surface grid distribution from the downwash study medium grid was used with a smaller minimum wall spacing. With maximum grid sizes exceeding 100 million nodes, the grid convergence study was particularly challenging for the node-based unstructured grid generators and flow solvers. At the time of the workshop, the super-fine grid with 105 million nodes and 600 million elements was the largest grid known to have been generated using VGRID. Some improvements to the FUN3D solver/grid processing efficiency and parallelization were shown which allowed for practical pre-processing and solution times on the largest unstructured-grid size requested for the workshop.

The FUN3D workshop results were computed using the full Navier-Stokes equations with the flux-differencesplitting scheme of Roe, the Venkatakrishnan flux-limiter, and the loosely-coupled S-A turbulence model. Good residual, force, and moment convergence was achieved for the all the workshop computations even on the super-fine grid size. Overall, the FUN3D results compared well with the structured-grid code CFL3D on the finer grid levels. For the constant lift grid convergence case, the convergence of total drag was approximately second-order on the finest three grids. The variation in total drag on the finest three grids was about 6 counts, and the variation on the finest two grids was 2 counts. At the finest grid levels, only small variations in wing and tail pressure distributions were seen with grid refinement. Similarly, a small wing/side-of-body separation also showed little variation at the finest grid levels. The FUN3D downwash study and Reynolds number study results compared well with the range of results shown in the DPW-IV workshop presentations. Additional comparisons of the FUN3D results will be made when the experimental data for the CRM and the statistical analysis of the workshop results are made publicly available.

\section{Acknowledgments}

The authors would like to thank Mr. Mark Chaffin of the Cessna Aircraft Company for providing the Gridtool restart files for use in the grid generation process.

\section{References}

\footnotetext{
${ }^{1}$ Anon., "Fourth AIAA Drag Prediction Workshop, San Antonio, TX," http://aaac.larc.nasa.gov/tsab/cfdlarc/aiaa-dpw, Dec. 2009.

${ }^{2}$ Levy, D. W., Zickuhr, T., Vassberg, J., Agrawal, S., Wahls, R. A., Pirzadeh, S., and Hemsch, M. J., "Data Summary from the First AIAA Computational Fluid Dynamics Drag Prediction Workshop," Journal of Aircraft, Vol. 40, No. 5, 2003, pp. 875-882, See also AIAA Paper 20020841 .
} 
${ }^{3}$ Laflin, K., Klausmeyer, S., Zickuhr, T., Vassberg, J., Wahls, R., Morrison, J., Brodersen, O., Rakowitz, M., Tinoco, E., and Godard, J.-L., "Data Summary from Second AIAA Computational Fluid Dynamics Drag Prediction Workshop," Journal of Aircraft, Vol. 42, No. 5, May 2005, pp. 1165-1178, See also AIAA Paper 2004-0555.

${ }^{4}$ Vassberg, J., Tinoco, E., Mani, M., Brodersen, O., Eisfeld, B., Wahls, R., Morrison, J., Zickuhr, T., Laflin, K., and Mavriplis, D., "Abridged Summary of the Third AIAA Computational Fluid Dynamics Drag Prediction Workshop," Journal of Aircraft, Vol. 45, No. 3, March 2008, pp. 781798, See also AIAA Paper 2007-0260.

${ }^{5}$ Vassberg, J. C., DeHaan, M. A., Rivers, S. M., and Wahls, R. A., "Development of a Common Research Model for Applied CFD Validation Studies," AIAA Paper 2008-6919, Aug. 2008.

${ }^{6}$ Rivers, M. and Dittberner, A., "Experimental Investigations of the NASA Common Research Model," AIAA Paper 2010-4218, June 2010.

${ }^{7}$ Morrison, J., "Statistical Analysis of CFD Solutions from the Fourth AIAA Drag Prediction Workshop," AIAA Paper 2010-4673, June 2010.

${ }^{8}$ Eliasson, P. and Peng, S., "Influence of Turbulence Modelling and Grid Resolution in Computations of the DPW-4 CRM Configuration," AIAA Paper 2010-1416, Jan. 2010.

${ }^{9}$ Hashimoto, A., Murakami, K., Aoyama, T., Yamamoto, K., Murayama, M., and Lahur, P. R., "Drag Prediction on NASA CRM Using Automatic Hexahedra Grid Generation,” AIAA Paper 2010-1417, Jan. 2010.

${ }^{10}$ Anderson, W. K. and Bonhaus, D. L., "An Implicit Upwind Algorithm for Computing Turbulent Flows on Unstructured Grids," Computers and Fluids, Vol. 23, No. 1, 1994, pp. 1-22.

${ }^{11}$ Anderson, W. K., Rausch, R. D., and Bonhaus, D. L., "Implicit/Multigrid Algorithms for Incompressible Turbulent Flows on Unstructured Grids," Journal of Computational Physics, Vol. 128, No. 2, 1996, pp. 391-408.

${ }^{12}$ Nielsen, E. J., Aerodynamic Design Sensitivities on an Unstructured Mesh Using the Navier-Stokes Equations and a Discrete Adjoint Formulation, Ph.D. thesis, Virginia Polytechnic Institute and State University, Blacksburg, VA, 1998.

${ }^{13}$ Sclafani, A. J., Vassberg, J. C., Rumsey, C. L., and DeHaan, M. A., "Drag Prediction for the NASA CRM Wing/Body/Tail Using CFL3D and OVERFLOW on an Overset Mesh," AIAA Paper 2010-4219, June 2010.

${ }^{14}$ Pirzadeh, S. Z., "Advanced Unstructured Grid Generation for Complex Aerodynamic Applications," AIAA Journal, Vol. 48, No. 5, May 2010, pp. 904-915, See also AIAA Paper 2008-7178.

${ }^{15}$ Pirzadeh, S. Z. and Frink, N. T., "Assessment of the Unstructured Grid Software TetrUSS for Drag Prediction of the DLR-F4 Configuration," AIAA Paper 2002-0839, Jan. 2002.

${ }^{16}$ Roe, P. L., "Approximate Riemann Solvers, Parameter Vectors, and Difference Schemes," Journal of Computational Physics, Vol. 43, 1981, pp. 357-372.

${ }^{17}$ Venkatakrishnan, V., "Convergence to Steady State Solutions of the Euler Equations on Unstructured Grids with Limiters," Journal of Computational Physics, Vol. 118, No. 1, 1995, pp. 120-130.

18 Anderson, W. K. and Bonhaus, D. L., "An Implicit Upwind Algorithm for Computing Turbulent Flow on Unstructured Grids," Computers and Fluids, Vol. 23, No. 1, Jan. 1994, pp. 1-21.

${ }^{19}$ Diskin, B., Thomas, J. L., Nielsen, E. J., and White, J. A., "Comparison of Node-Centered and Cell-Centered Unstructured Finite-Volume Discretizations Part I: Viscous Fluxes," AIAA Paper 2009-0597, Jan. 2009.

${ }^{20}$ Nielsen, E. J., Lu, J., Park, M. A., and Darmofal, D. L., "An Implicit, Exact Dual Adjoint Solution Method for Turbulent Flows on Unstructured Grids," Computers and Fluids, Vol. 33, No. 9, 2004, pp. 1131-1155, See also AIAA Paper 2003-0272.

${ }^{21}$ Spalart, P. R. and Allmaras, S. R., "A One-Equation Turbulence Model for Aerodynamic Flows," La Recherche Aerospatiale, , No. 1, 1994, pp. 5-21, See also AIAA Paper 92-0429.

${ }^{22}$ Menter, F. R., "Two-Equation Eddy-Viscosity Turbulence Models for Engineering Applications," AIAA Journal, Vol. 32, No. 8, 1994, pp. $1598-1605$.

${ }^{23}$ Krist, S. L., Biedron, R. T., and Rumsey, C. L., CFL3D User's Manual (Version 5.0), NASA TM-1998-208444, June 1998.

${ }^{24}$ Sclafani, A. J., Vassberg, J. C., Harrison, N. A., DeHaan, M. A., Rumsey, C. L., Rivers, S. M., and Morrison, J. H., "CFL3D/OVERFLOW Results for DLR-F6 Wing/Body and Drag Prediction Workshop Wing," Journal of Aircraft, Vol. 45, No. 3, May 2008, pp. $762-780$.

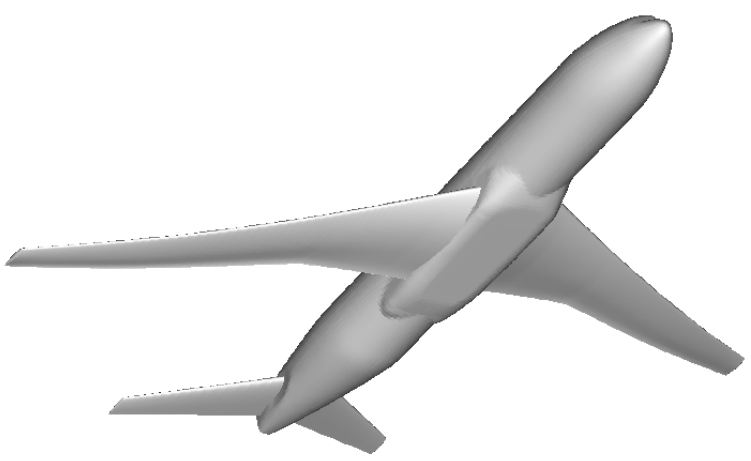

Figure 1. NASA Common Research Model wing/body/tail configuration. 

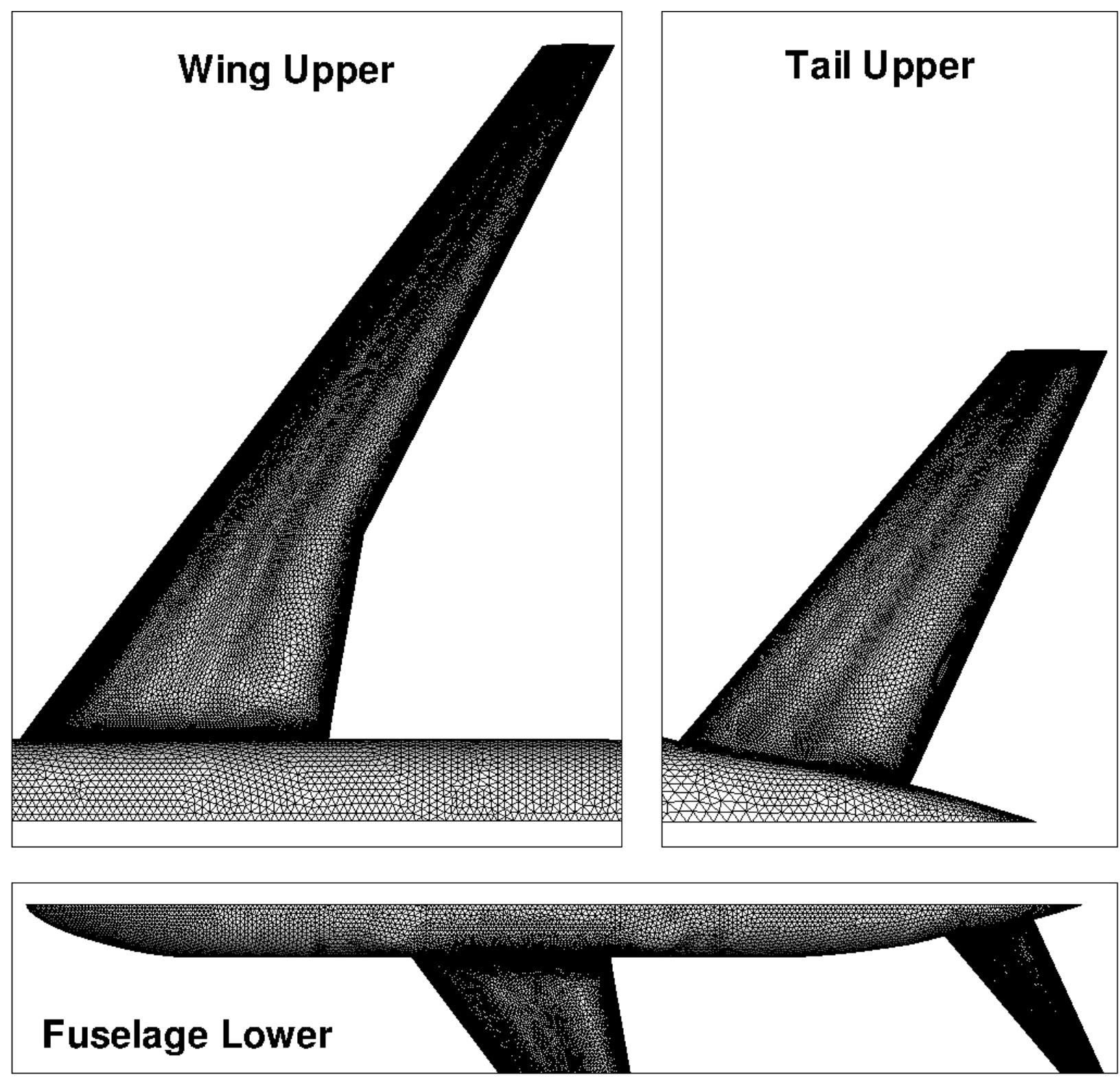

Figure 2. Surface grid for the CRM wing/body/tail medium-sized grid (10.3 million nodes). 


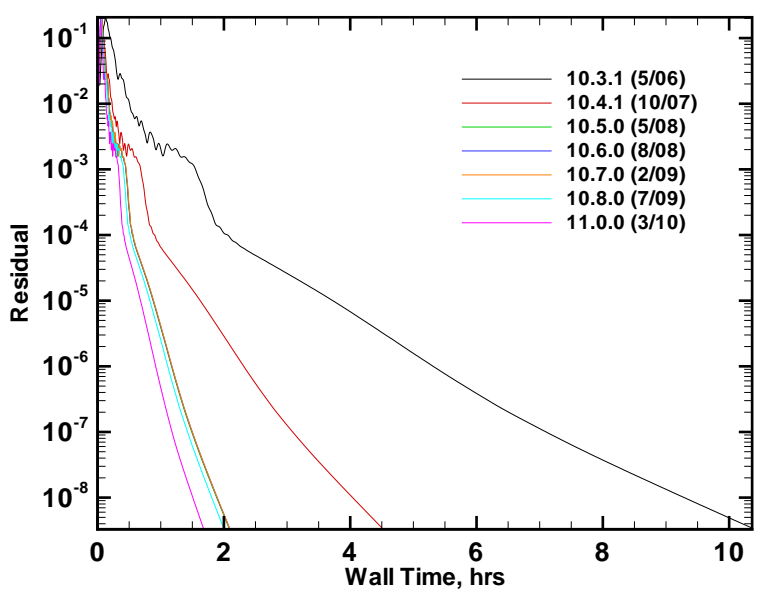

(a) Computational Timing

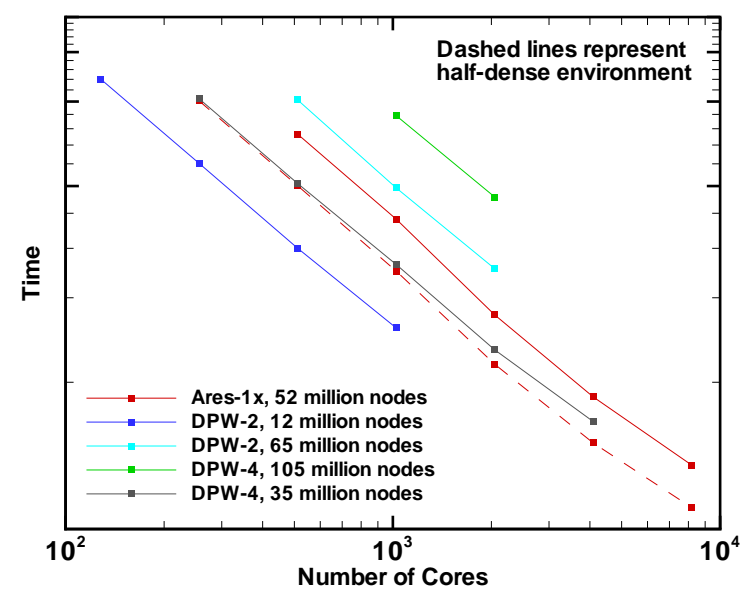

(b) Parallel scalability

Figure 3. FUN3D computational performance. 


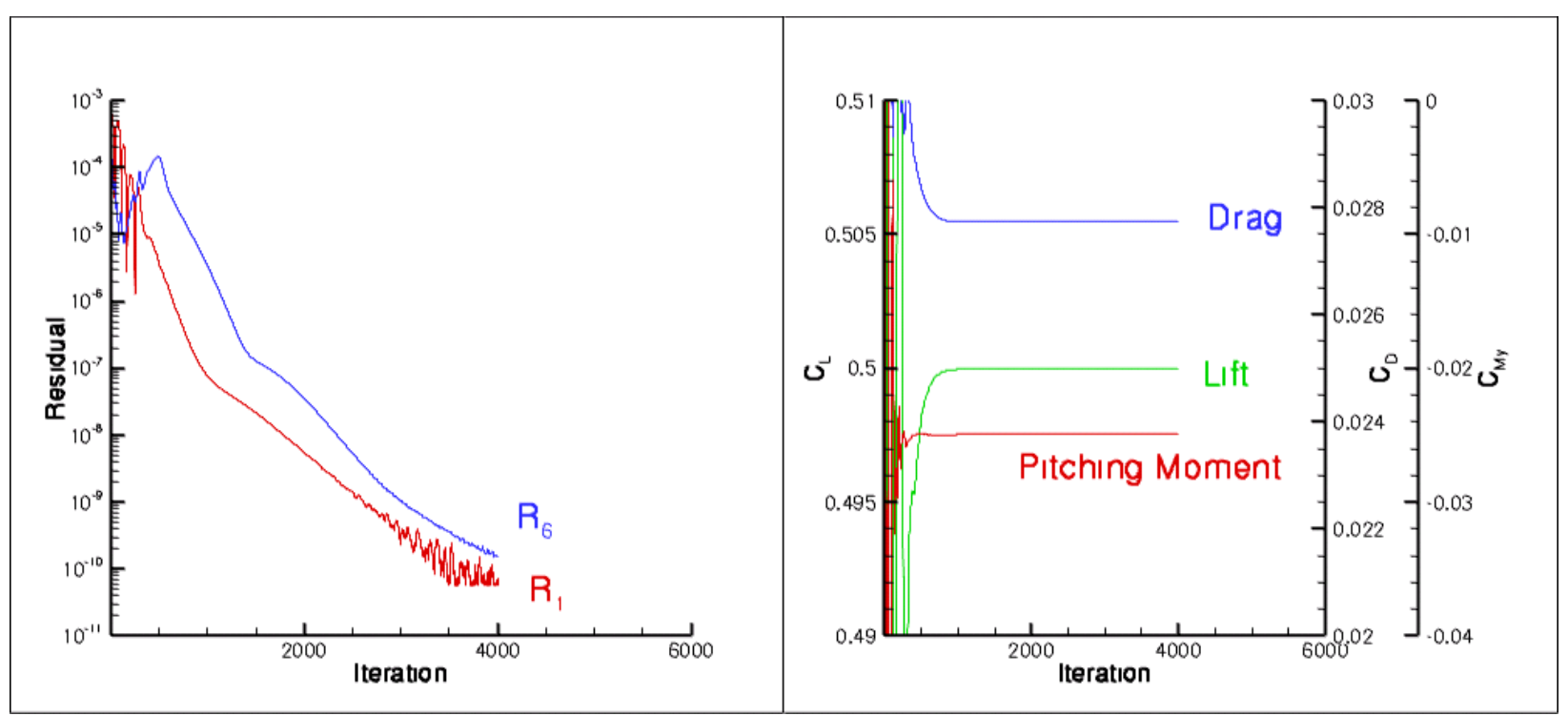

(a) Medium grid (10.3 million nodes)
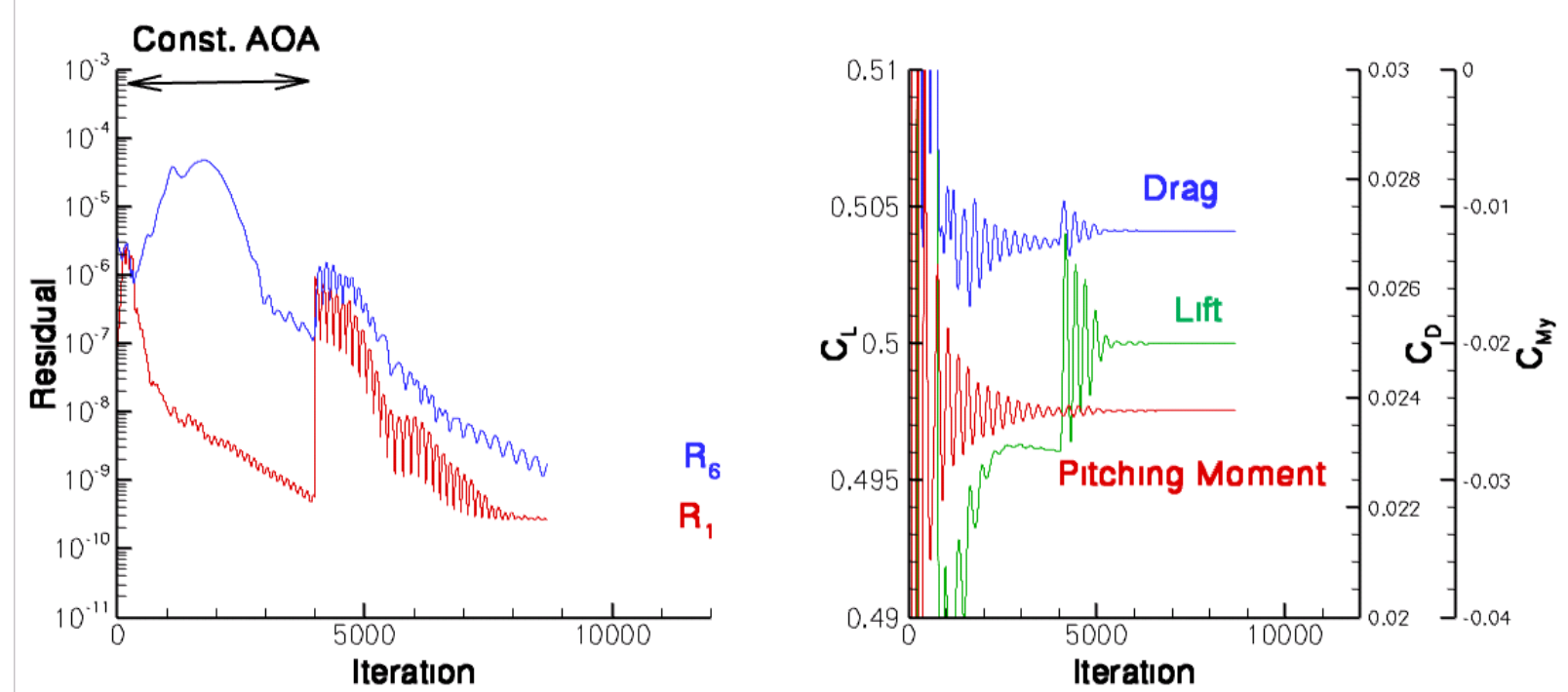

(b) Super-Fine grid (105 million nodes)

Figure 4. Non-linear solution residual, force, and moment convergence history for the CRM wing/body/tail. 

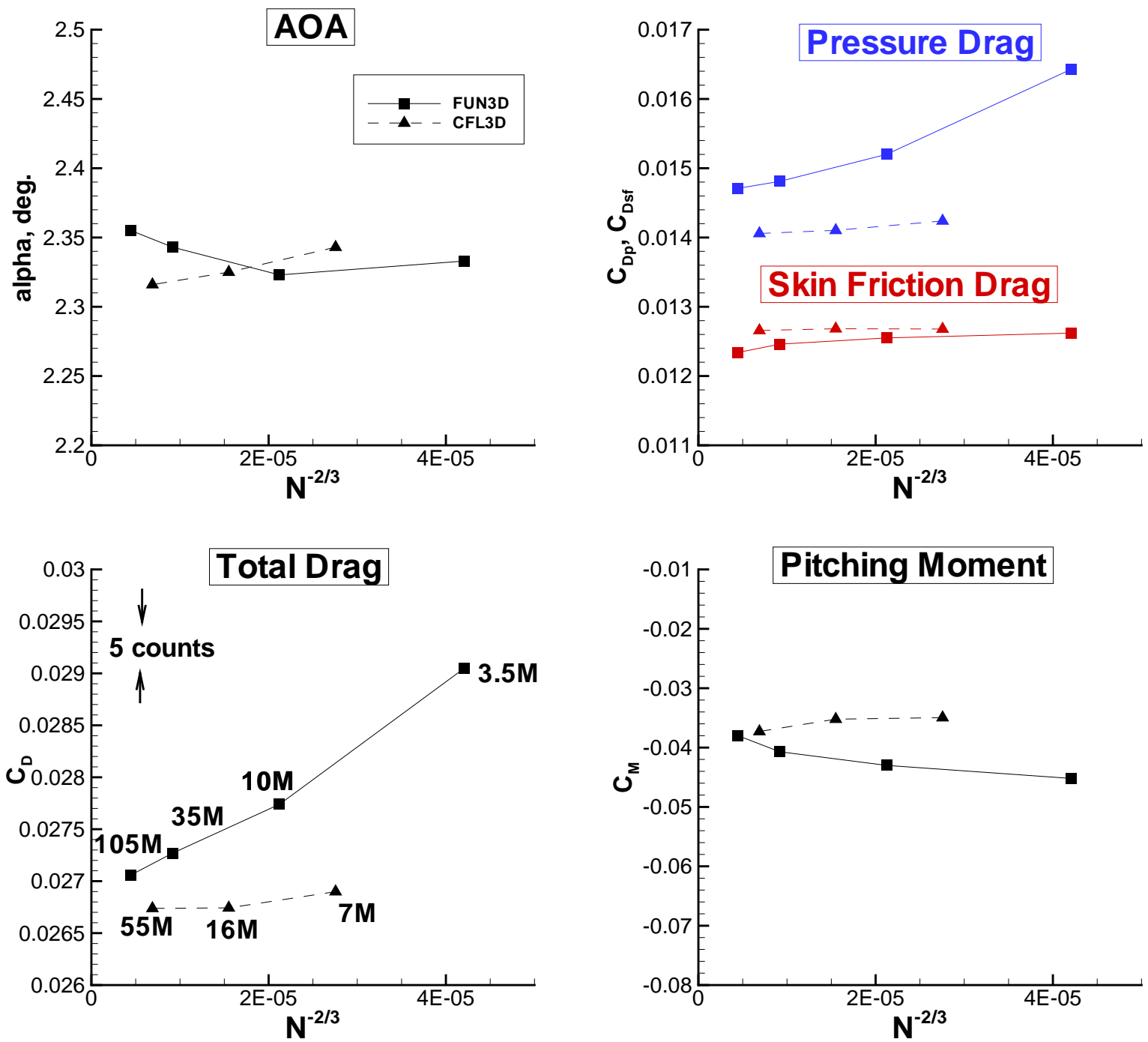

Figure 5. Grid convergence of CRM total forces and moment at $M_{\infty}=0.85, R e_{c}=5 \times 10^{6}$. 


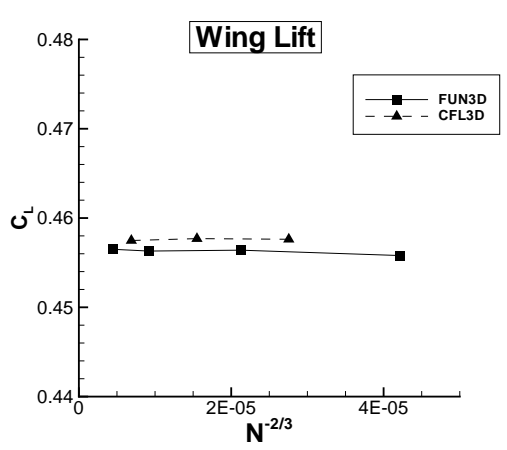

(a) Wing Lift

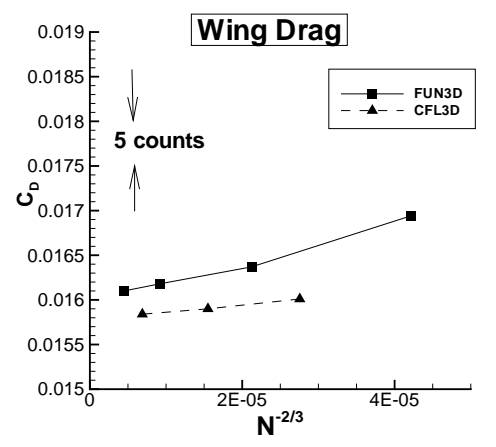

(d) Wing Drag

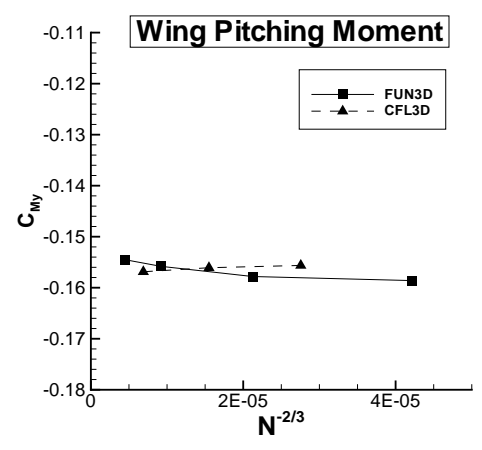

(g) Wing Pitching Moment

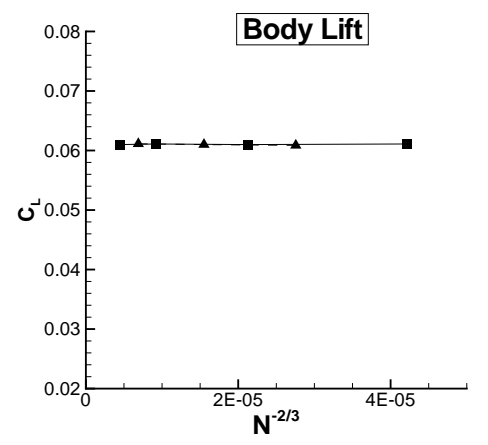

(b) Body Lift

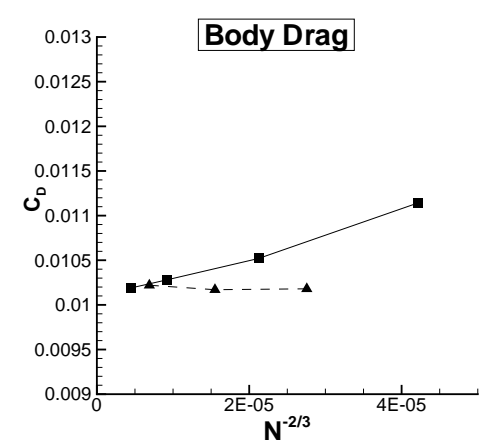

(e) Body Drag

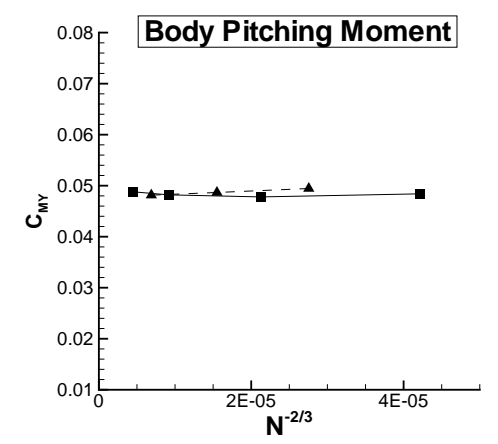

(h) Body Pitching Moment

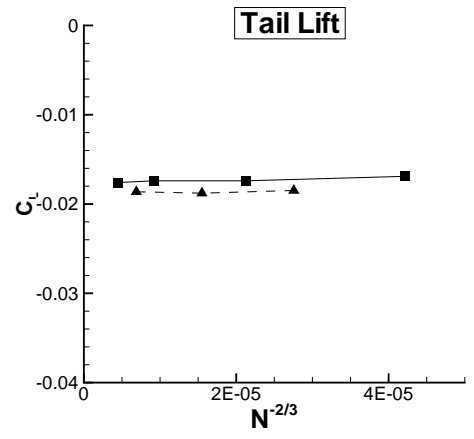

(c) Tail Lift

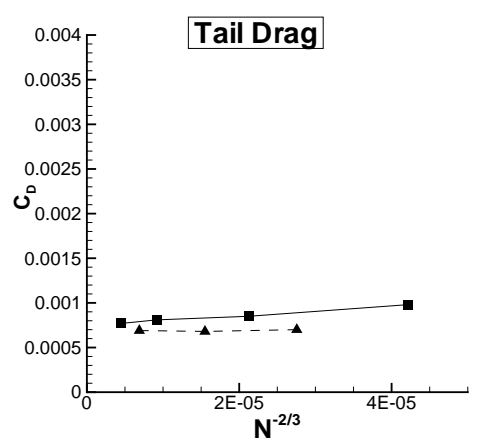

(f) Tail Drag

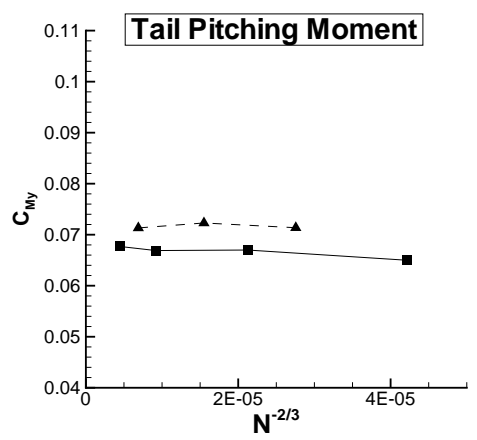

(i) Tail Pitching Moment

Figure 6. Grid convergence of CRM component forces and moment at $M_{\infty}=0.85, R e_{c}=5 \times 10^{6}$. 

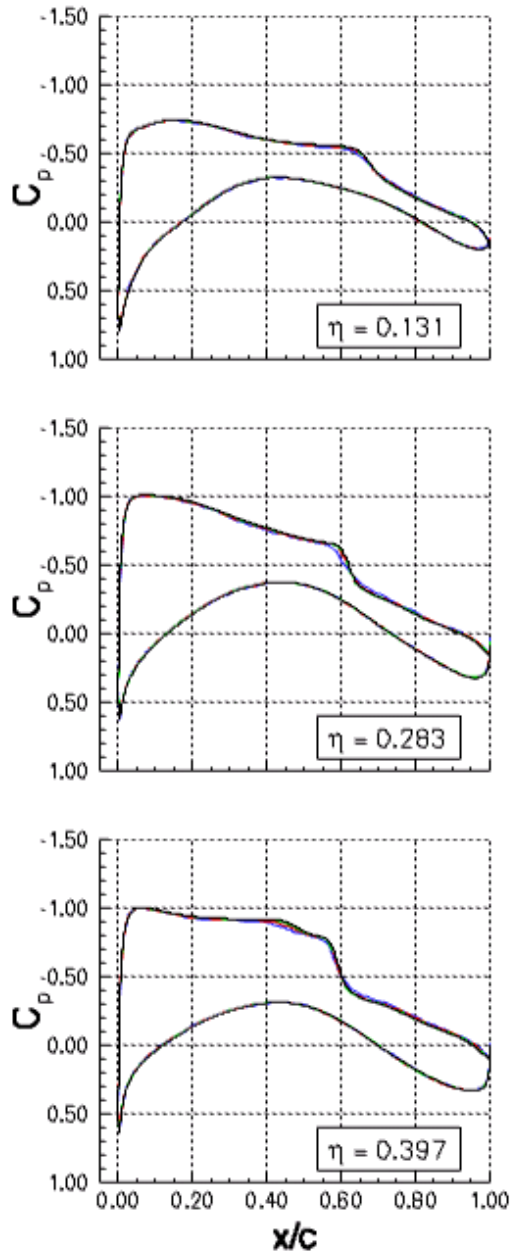
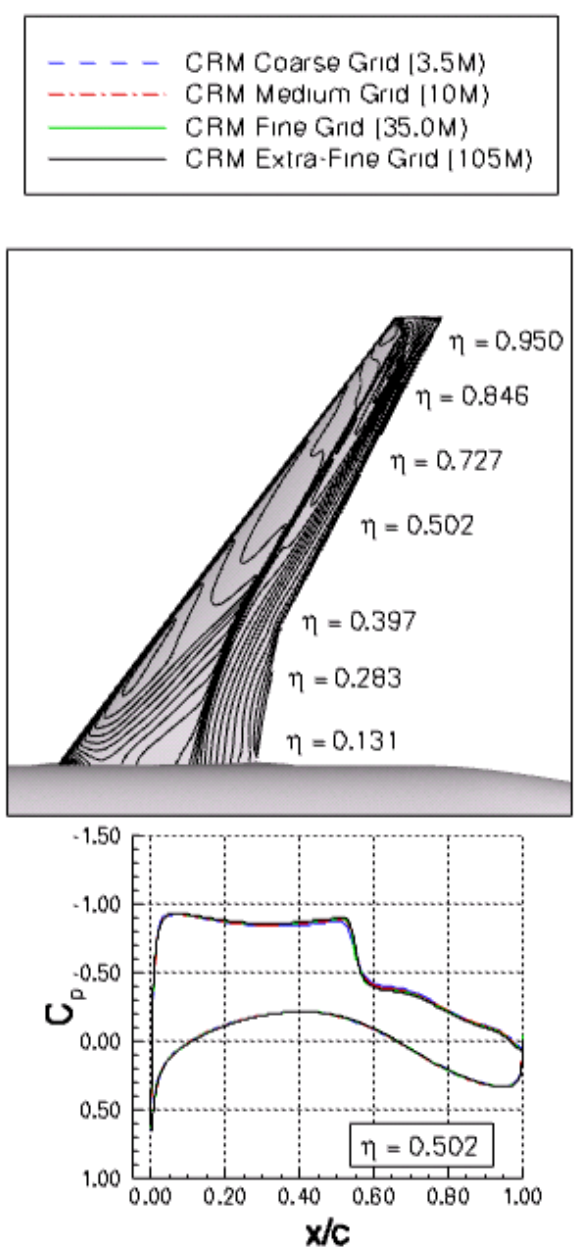
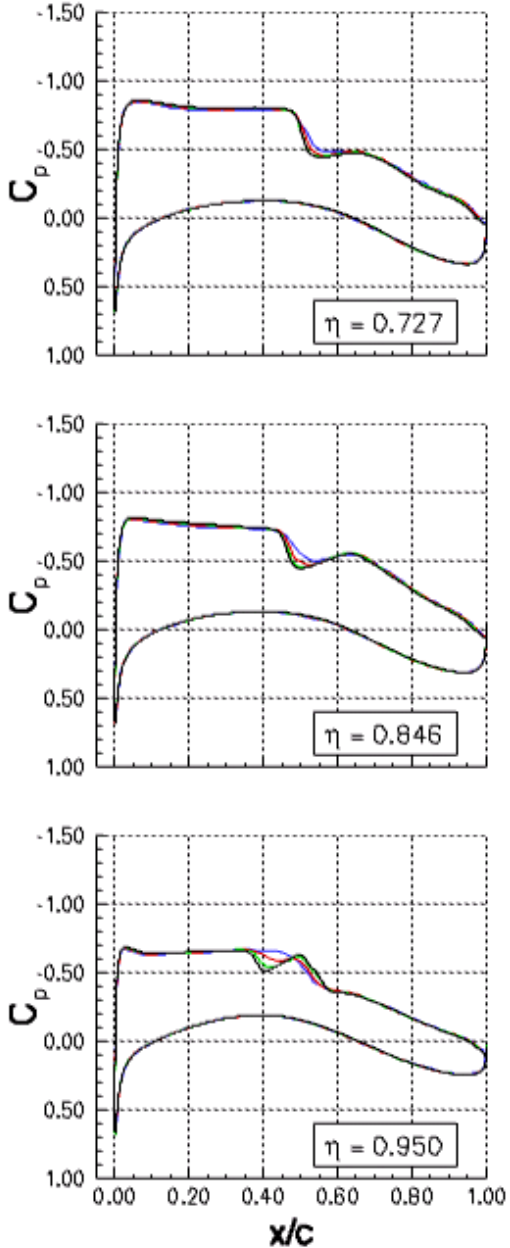

Figure 7. Grid convergence of CRM wing chordwise pressure distributions at $M_{\infty}=0.85, R e_{c}=5 \times 10^{6}$ (surface pressure contours from fine grid solution). 

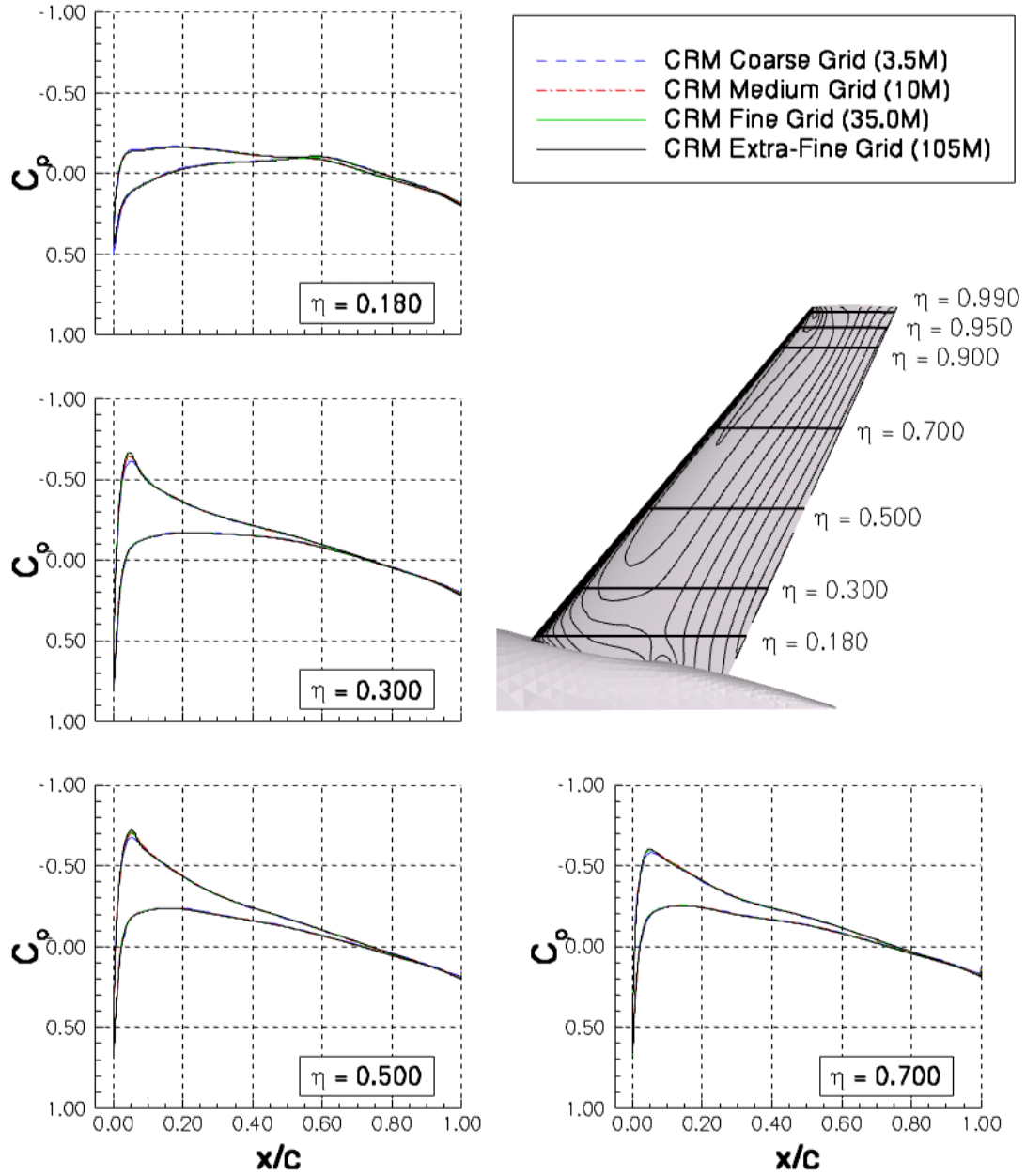
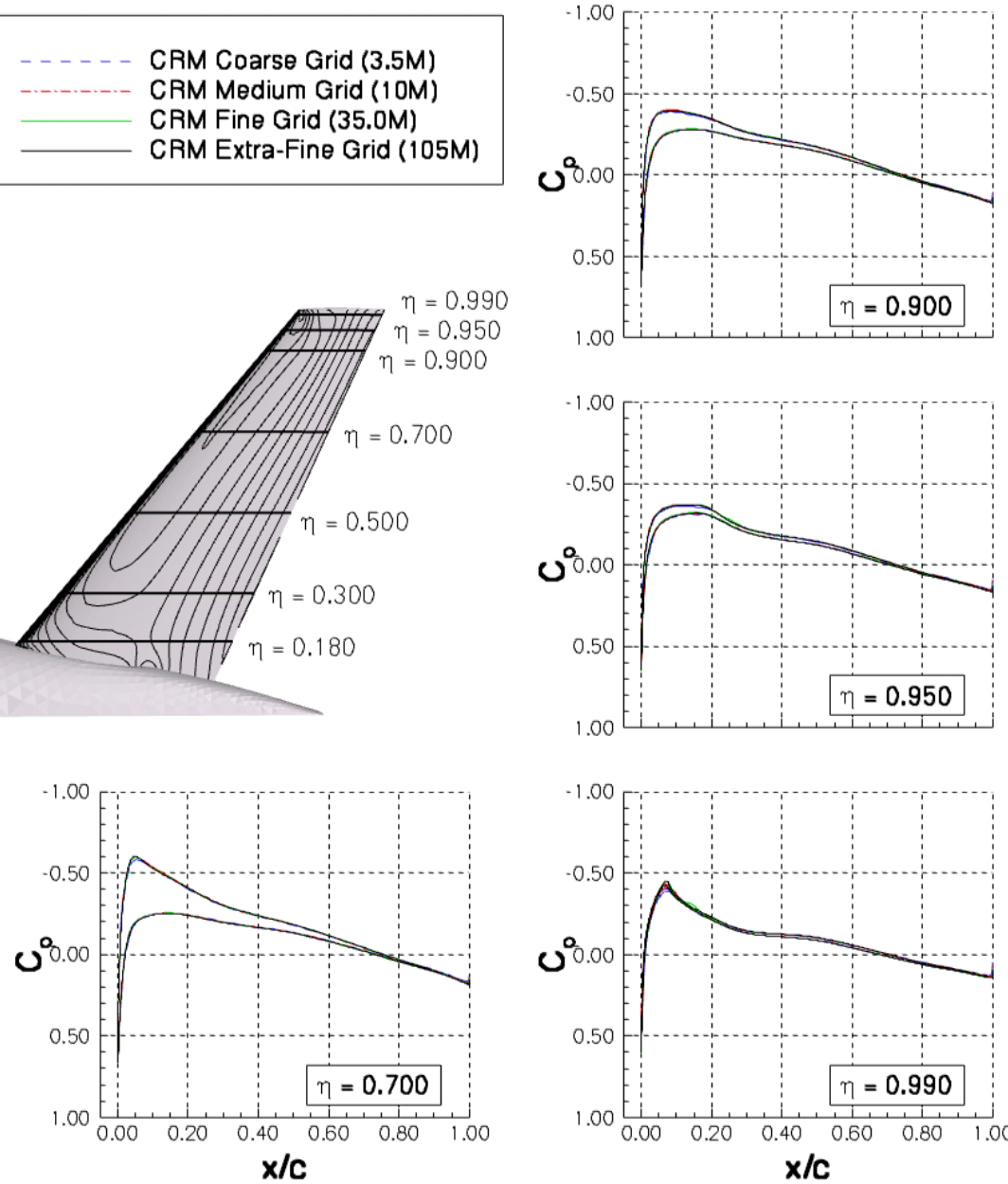

Figure 8. Grid convergence of CRM tail chordwise pressure distributions at $M_{\infty}=0.85, R e_{c}=5 \times 10^{6}$ (surface pressure contours from fine grid solution). 

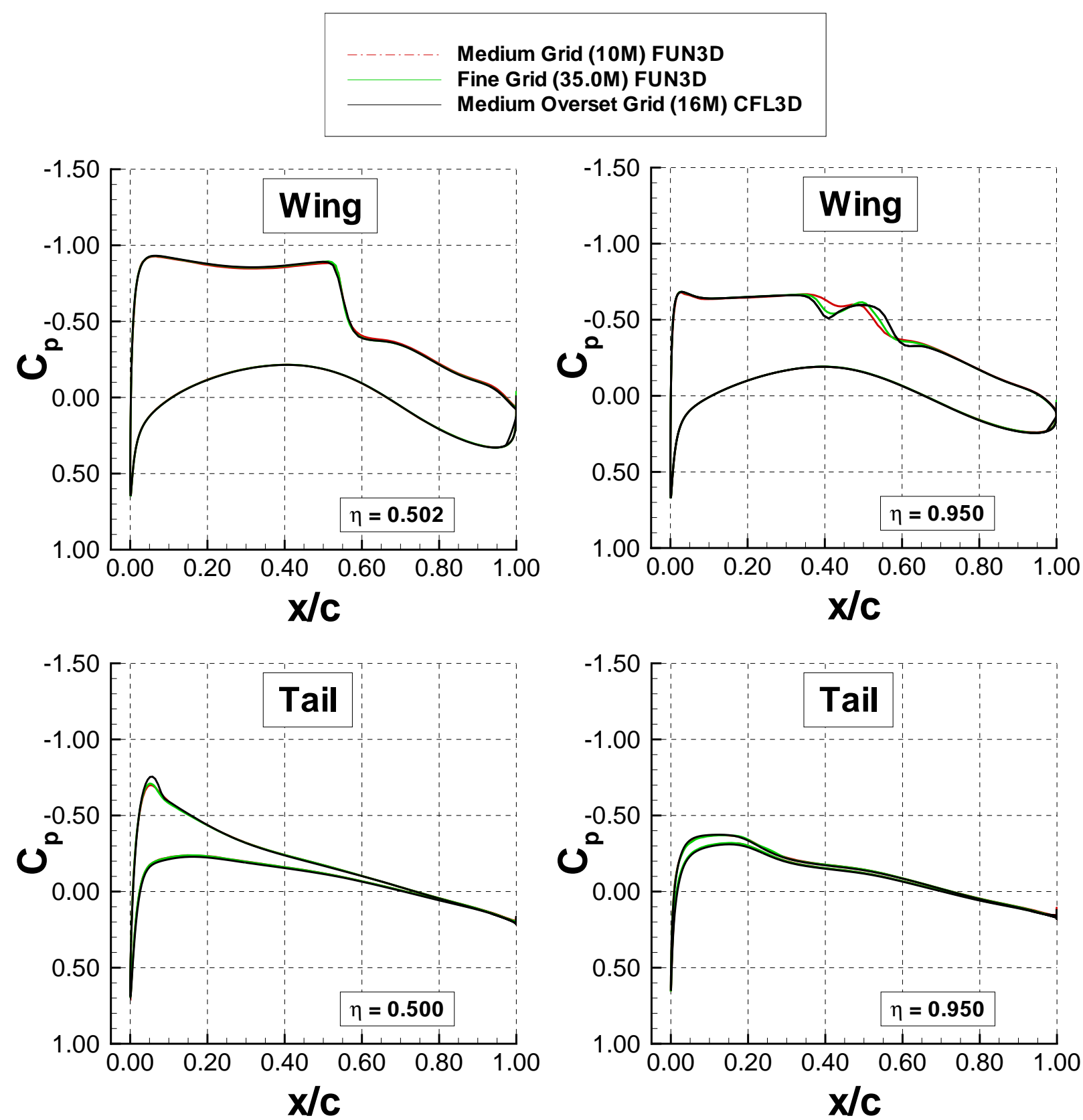

Figure 9. Comparison of FUN3D and CFL3D CRM wing/tail chordwise pressure distributions at $M_{\infty}=0.85, R e_{c}=5 \times 10^{6}$. 


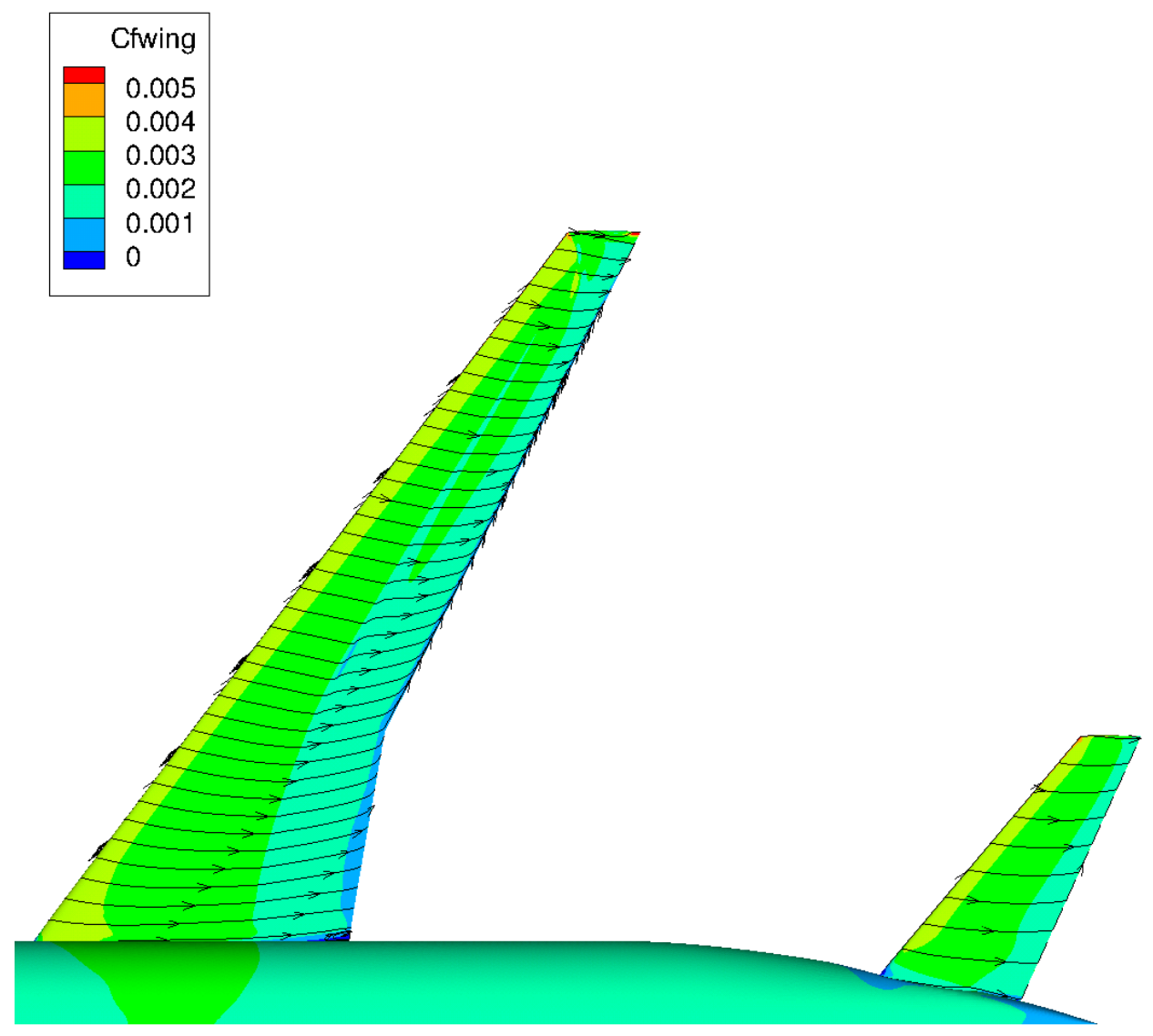

Figure 10. CRM fine grid ( 35 million nodes) skin friction coefficient and surface restricted streamlines at $M_{\infty}=0.85, R_{c}=5 \times 10^{6}$. 


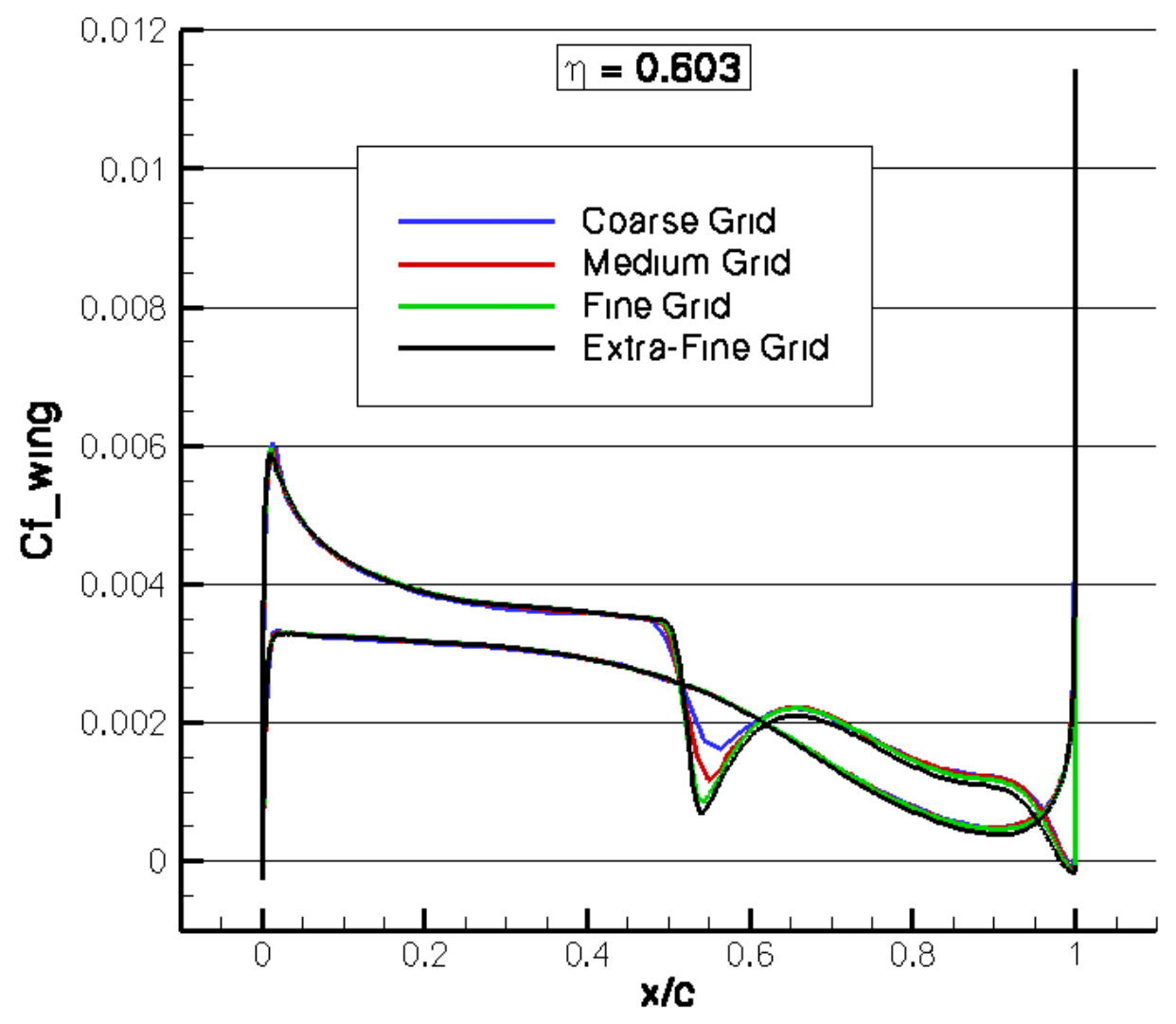

Figure 11. Grid convergence of CRM wing chordwise skin friction distribution at $M_{\infty}=0.85, R e_{c}=5 \times 10^{6}$. 


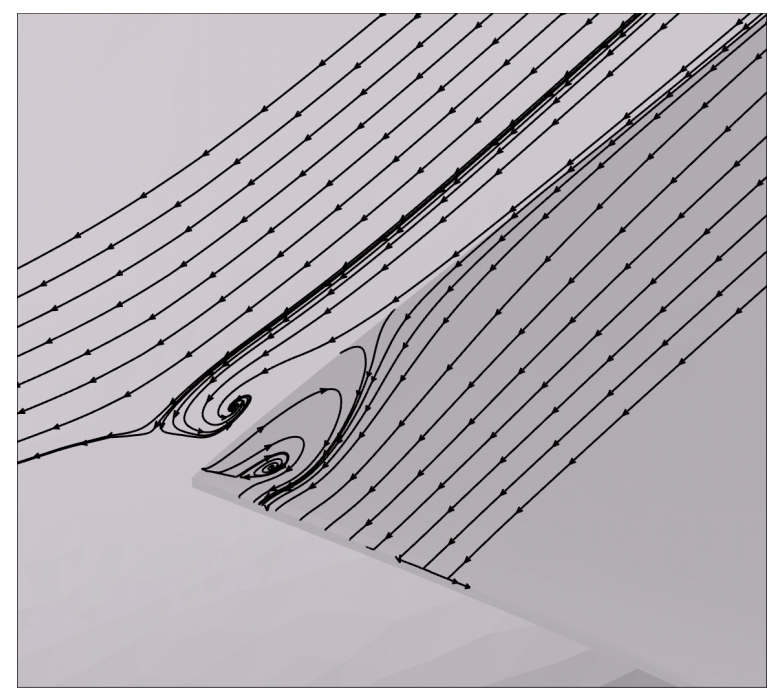

(a) Coarse Grid

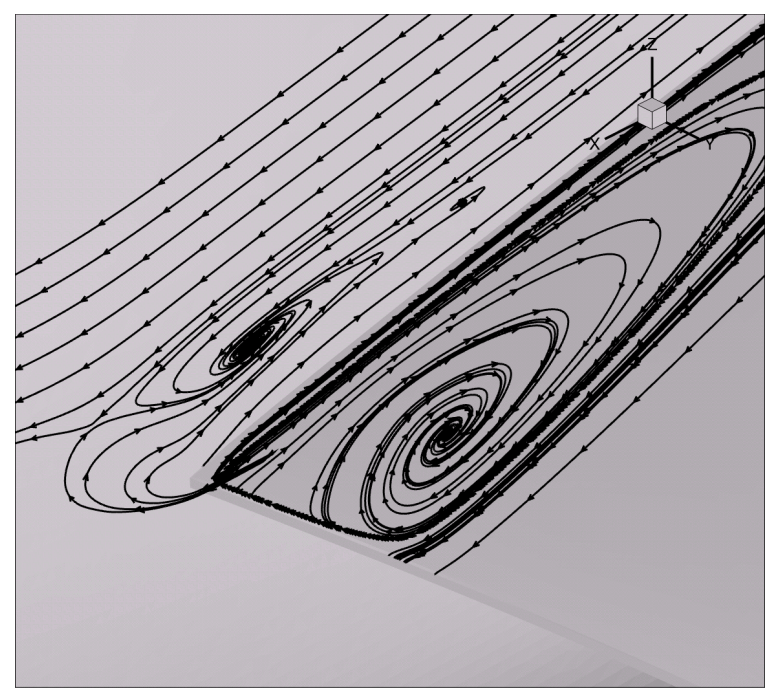

(c) Fine Grid

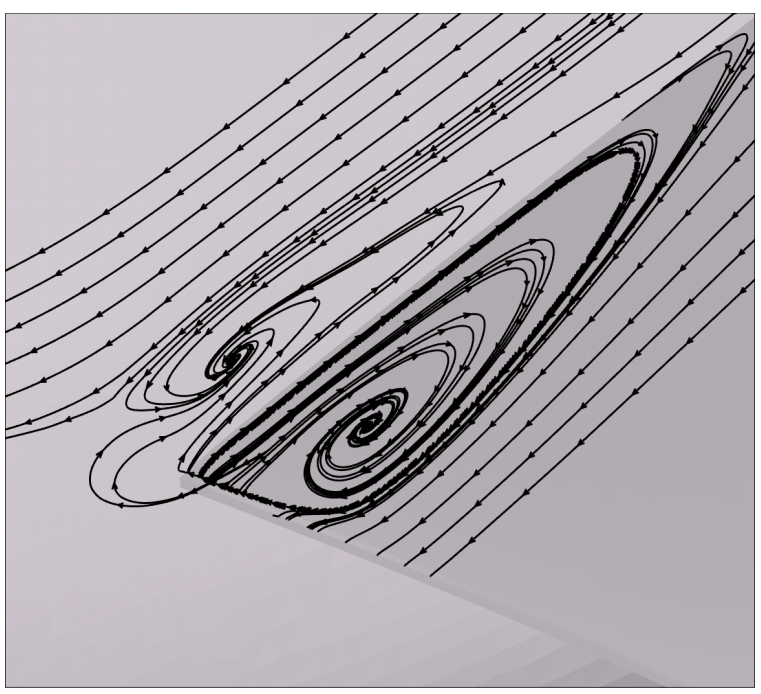

(b) Medium Grid

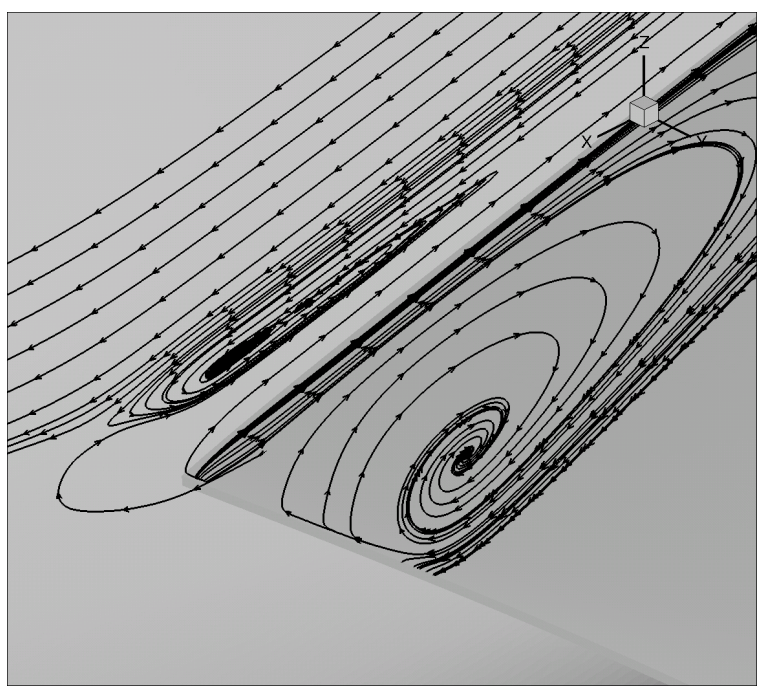

(d) Extra-Fine Grid

Figure 12. Effect of grid density on the CRM $\left(C_{L}=0.5\right)$ trailing edge wing-root juncture separation. 

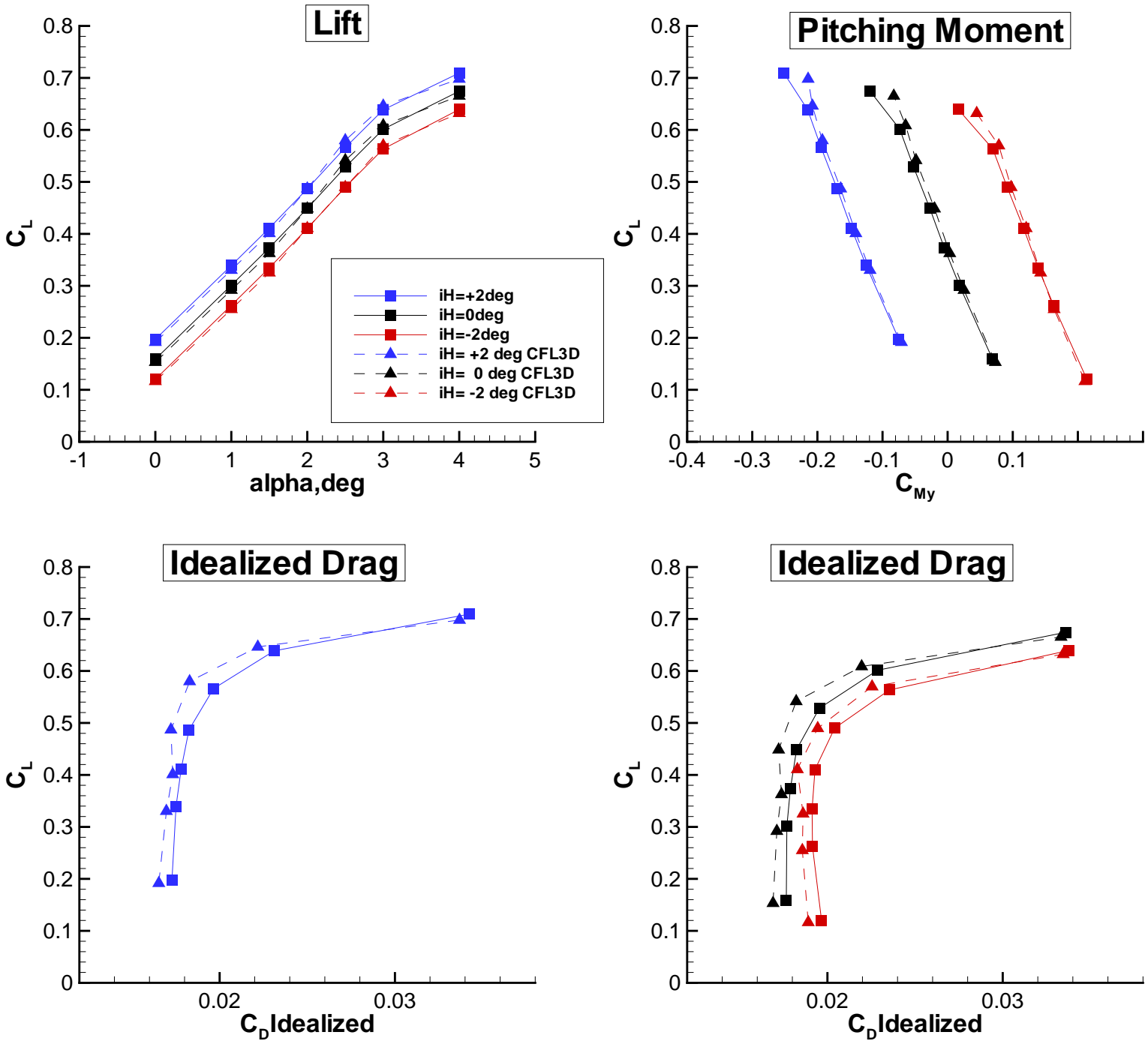

Figure 13. Effect of tail incidence on CRM forces and moments at $M_{\infty}=0.85, \operatorname{Re}_{c}=5 \times 10^{6}$. 

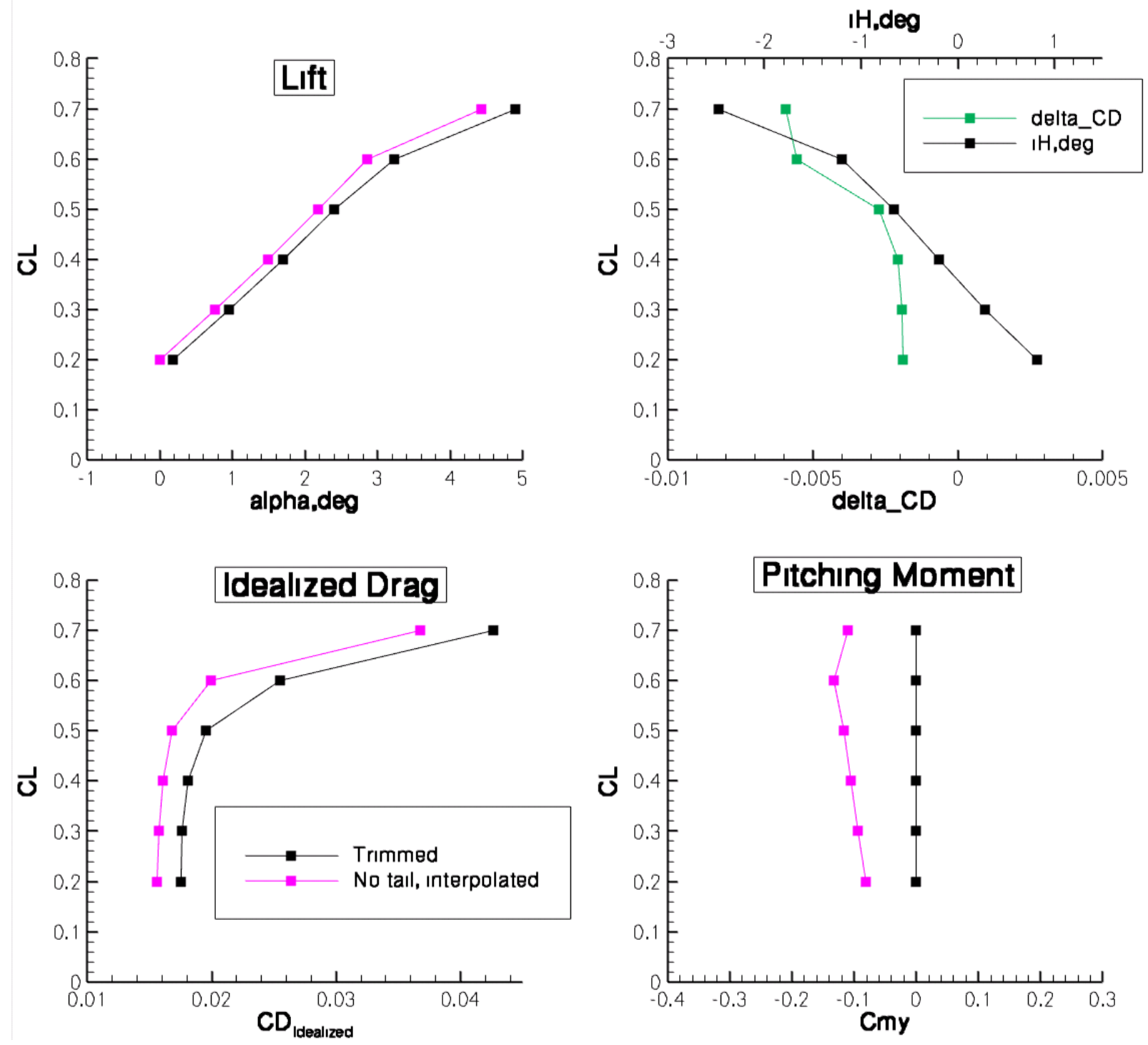

Figure 14. Comparison of the CRM tail-off and trimmed tail-on forces and moments at $M_{\infty}=0.85, R e_{c}=5 \times 10^{6}$. 